\title{
On Mesh Refinement and Accuracy of Numerical Solutions
}

\author{
Hong Zhou ${ }^{1}$, Maria Peters ${ }^{1}$ and Adriaan van Oasterom ${ }^{2}$ \\ ${ }^{1}$ Department of Low Temperature, University of Twente \\ ${ }^{2}$ Laboratory of Medical Physics and Biophysics, University of Nijmegen
}

\begin{abstract}
This paper investigates mesh refinement and its relation with the accuracy of the boundary element method (BEM) and the finite element method (FEM). To this end an isotropic homogeneous spherical volume conductor, for which the analytical solution is available, was used. The numerical results obtained with the BEM and FEM were compared with the results of the analytical solution. The results show that the accuracy of the numerical solutions is improved by enriching a mesh only if the enriched mesh not only incorporates a greater number of nodes but also follows more closely the actual geometry of the volume conductor involved.
\end{abstract}

\section{Introduction}

With the advance of modern computer technology numerical solution methods such as the boundary element method and the finite element method have been widely used in the studies of electrophysiology. One of the concerns in the application of these numerical solution methods is their numerical accuracy. To assess the quality of the numerical solution of the BEM and FEM obtained with a given mesh, different error indicators have been proposed and adaptively mesh refinement have been performed to increase the resolution of the numerical solutions $[1,2,3]$.

In this paper the mesh refinement and its relation with the accuracy of the numerical solutions will be discussed. It will be demonstrated by numerical examples that, in certain circumstances, simple mesh refinement can lead to the decrease in the accuracy of the numerical solutions.

\section{Mesh Refinement and Accuracy}

The electric potential inside a homogeneous volume conductor $V$ enclosed by a surface $S$ due to an internal current source is described by Poisson's equation. In a Cartesian coordinate system $x_{1}, x_{2}, x_{3}$ this reads

$$
\nabla \cdot(\overrightarrow{\vec{\sigma}} \nabla \Phi)=-i_{v}(\vec{x}) \text {, }
$$

where $\Phi(\vec{x})$ is the potential field, $\overrightarrow{\vec{\sigma}}$ is the conductivity tensor, and $i_{v}\left(\vec{x}^{\prime}\right)$ is the impressed current source volume density. The boundary condition implied by the bounding surface $S$ of the bounded volume conductor is

$$
\vec{n} \cdot(\overrightarrow{\vec{\sigma}} \nabla \Phi)=0,
$$

in which $\vec{n}$ is the unit outward normal vector on the surface $S$.

As is well known this problem can be solved numerically by either the boundary element method or the finite element method. In the BEM the boundary surface $S$ is approximately represented by a triangulated surface $S_{d}$. The potential $\Phi_{S_{d}}$ is then assumed to be a constant for which the potential at the center of mass of the triangles is taken [4]. In the FEM the 3-D problem domain is approximately represented by a set of tetrahedron or hexahedron elements $V_{d}$ that are connected to each other in such \& way that there is neither overlap nor gap among them. Within each element the solution $\Phi_{V_{d}}$ is assumed to be a polynomial function, having the potentials at the nodes as its parameters.

There are two different kinds of approximations involved in these numerical methods. One is the approximation of the solution domain: the approximation of $S$ by $S_{d}$ for the BEM and the approximation, of $V$ by $V_{d}$ for the FEM. The other is the approximation of the second order differentiable solution: the approximation of $\Phi$ by a piecewise constant solution $\Phi_{S_{d}}$ for the BEM and the approximation of $\Phi$ by a piecewise linear or tri-linear solution $\Phi_{V_{d}}$ for the FEM.

Once the numerical solution $\Phi_{S_{d}}$ or $\Phi_{V_{d}}$ is obtained certain criteria can be established to judge the fineness of the elements $[1,5]$. The elements where the criterion is not satisfied are refined by adding new nodes. As the number of elements increases, the numerical solution converges. The straightforward element enrichment, however, does not improve per se the approximation of the boundary surface $S$, or the approximation of the solution domain $V$ if new nodes are merely added on surface elements. The numerical solution may converge to the true solution on $S_{d}$ for the BEM or in $V_{d}$ for the FEM, but not the true solution on $S$ or $V$.

The relative difference of the peak to peak value between the analytical solution and any of the numerical solutions considered, defined as

$$
\epsilon_{r}=\frac{\left|T\left(\Phi_{a n l}\right)-T\left(\Phi_{n u a}\right)\right|}{T\left(\Phi_{a n l}\right)}
$$

is used to asses the numerical accuracy, where $T(\Phi)=$ $\Phi_{\max }-\Phi_{\min }$. 


\section{Examples}

A spherical volume conductor with radius 3 and conductivity 1 was used in numerical computations. The origin of the coordinate system is at the center of the sphere with $z$-axis upwards. A unit z-oriented current dipole located at $(0,0,1)$ was used as the source of the electric potential.

For the computation of the BEM a coarse triangulated surface with 48 triangles and 26 vertexes as shown in Fig. $2 b$ was first made to approximate the surface of the sphere. Fig. 2c shows a triangulated surface with 192 triangles and 98 vertexes which was made by dividing each triangle of the coarse triangulated surface into 4 smaller triangles. Note the new vertexes are all located on the coarse triangulated surface, i.e., the coarse triangulated surface is embedded in the fine triangulated surface. Fig. $2 d$ shows a triangulated surface that is made by projecting the newly introduced vertexes of the surface shown in Fig. $2 c$ onto the surface of the sphere.

Fig. 1a shows the potential distribution of the surface of the sphere computed by means of the analytical solution. Fig. 1b, Fig. 1c and Fig. 1d show the results of the BEM, respectively, besed on the triangulated surfaces show $n$ in Fig. 2b, Fig. 2c and Fig. 2d. The relative errors of these results are $0.86 \%, 15.4 \%$ and $0.38 \%$ respectively.

Similar computations were carried out with the FEM. Fig. $4 \mathrm{~b}$ shows the perspective view of the surface elements of a coarse hexahedron mesh with 56 elements and 79 nodes. Fig. 4c shows a refined hexshedron mesh with 448 elements and 529 nodes which is generated by dividing each element of the coarse mesh shown in Fig. $4 \mathrm{~b}$ into 8 elements. Fig. $4 d$ shows a mesh that is made by projecting newly introduced surface nodes of the refined mesh onto the surface of the sphere.

Fig. 3a shows the potential distribution of the surface of the sphere computed by means of the analytical solution. Fig. 3b, Fig. 3c and Fig. 3d show the results of the FEM, respectively, based on the hexahedron mesh shown in Fig. $4 \mathrm{~b}$, Fig. $4 \mathrm{c}$ and Fig. $4 \mathrm{~d}$. The relative errors of these results are $3.0 \%, 7.1 \%$ and $2.3 \%$ respectively.

\section{Discussion}

The results show that the straightforward element enrichment, which does not improve the approximation of the geometry of the solution domain, does not self-evidently improve the numerical accuracy of the BEM and FEM, even though it does increase the resolution of the numerical solution. The error indicators that are commonly used are certainly appropriate for judging the fineness of the elements of a mesh. They do not, however, always reflect the accuracy of the numerical solutions. The results also show that the accuracy of the numerical solution is increased by improving the approximation of the geometry of the solution domain in the element enrichment. For the domain of irregular geometry, therefore, it is necessary to apply interpolation technique to make sure that the newly introduced vertexes in the $B E M$, or the newly introduced nodes which are belong to the boundary surface or interfaces bounding different compartments in the FEM, are located on the interpolated boundary surface or interfaces.

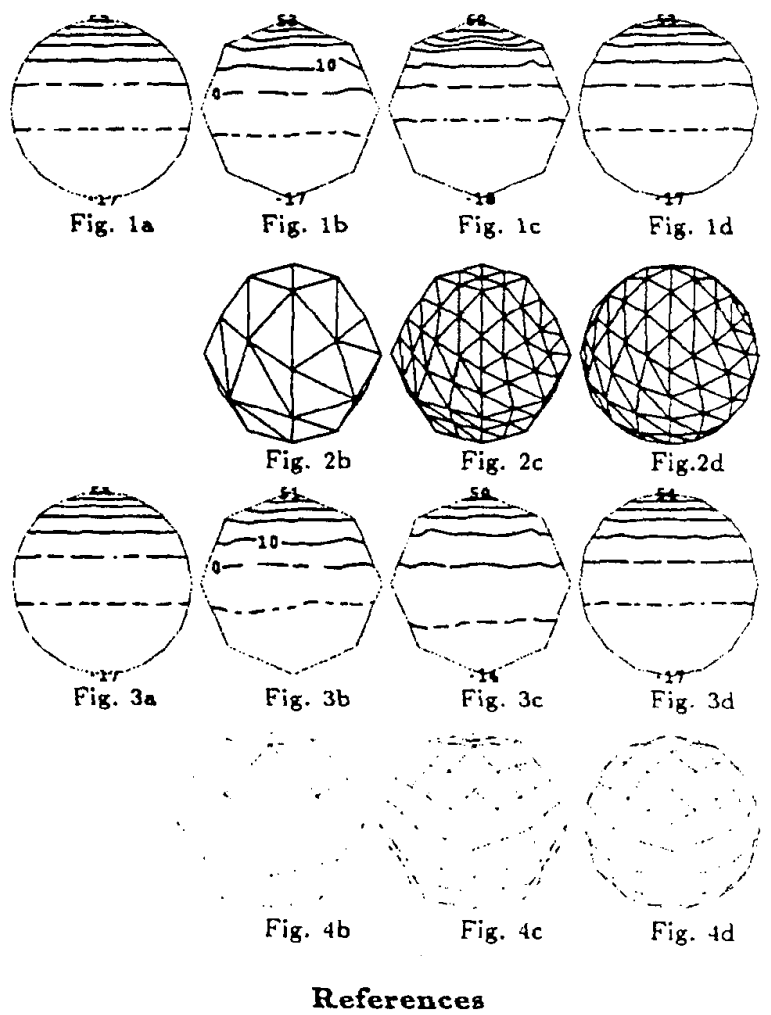

[1] Meijs JWH, Weier OW, Peters MJ, and van Oosterom $A$. On the numerical accuracy of the boundary element method. IEEE Trans. Biomed. Eng., 1989, BME-36:1038-1049.

[2] Miller CE and Henriquez CS. Finite element analysis of bioelectric phenomena. CRC Critical Review in Biomed. Eng., 1990, 18:207-233.

[3] Schmidt J, Eason J, and Pilkington T. Adaptive grid generation. In Plonsey $\mathrm{R}$, editor, Proc. 14th conf. of the IEEE/EMBS, page 593, IEEE Publishing Services, New York, 1992.

[4] Barr RC, Ramsey M, and Spach MS. Relating epicardial to body surface potentials by means of transfer coefficients based on geometry measurements. IEEE Trans. Biomed. Eng., 1977, BME-24:1-11.

[5] Eason J, Schmidt J, and Pilkington T. Finite element error estimates and adaptive grid refinement. In Plonsey $\mathrm{R}$, editor, Proc. 14th conf. of the $I E E E / E M B S$, pages 594-595, IEEE Publishing Services, New York, 1992. 